This item was submitted to Loughborough's Research Repository by the author.

Items in Figshare are protected by copyright, with all rights reserved, unless otherwise indicated.

\title{
Extending a Theorem of Bergweiler and Langley concerning nonvanishing
} derivatives

PLEASE CITE THE PUBLISHED VERSION

LICENCE

CC BY-NC-ND 4.0

REPOSITORY RECORD

Clifford, E.F.. 2019. "Extending a Theorem of Bergweiler and Langley Concerning Nonvanishing Derivatives". figshare. https://hdl.handle.net/2134/375. 


\title{
Extending a Theorem of Bergweiler and Langley Concerning Nonvanishing Derivatives
}

\author{
E. F. Clifford
}

\begin{abstract}
We consider the differential operator $\Lambda_{k}$ defined by

$$
\Lambda_{k}(y)=\Psi_{k}(y)+a_{k-1} \Psi_{k-1}(y)+\ldots+a_{1} \Psi_{1}(y)+a_{0},
$$

where $a_{0}, \ldots, a_{k-1}$ are analytic functions of restricted growth and $\Psi_{k}(y)$ is a differential operator defined by $\Psi_{1}(y)=y$ and $\Psi_{k+1}(y)=y \Psi_{k}(y)+\left(\Psi_{k}(y)\right)^{\prime}$ for $k \in \mathbb{N}$. We suppose that $k \geq 3$, that $F$ is a meromorphic function on an annulus $\mathcal{A}\left(r_{0}\right)$, and that $\Lambda_{k}(F)$ has all its zeros on a set $E$ such that $E$ has no limit point in $\mathcal{A}\left(r_{0}\right)$. We suppose also that all simple poles $a$ of $F$ in $\mathcal{A}\left(r_{0}\right) \backslash E$ have $\operatorname{Res}(F, a) \notin\{1, \ldots, k-1\}$. We then deduce that $F$ is a function of restricted growth in the Nevanlinna sense. This extends a theorem of Bergweiler and Langley [1]. We show also that this result does not hold for $a_{0}, \ldots, a_{k-1}$ meromorphic functions. ${ }^{1}$
\end{abstract}

Mathematics Subject Classification (1991): 30D30, 30D35

Keywords: meromorphic functions, Nevanlinna theory.

\section{Introduction}

In [1], Bergweiler and Langley define a differential operator $\Psi_{k}(F)$ for $k \in \mathbb{N}$ by

$$
\Psi_{1}(F)=F, \quad \Psi_{k+1}(F)=F \Psi_{k}(F)+\left(\Psi_{k}(F)\right)^{\prime},
$$

for which we have the following lemma.

Lemma 1.1 ([1]) Let $f$ be meromorphic on a domain $\Omega$ and let $F=f^{\prime} / f$. Then for each $k \in \mathbb{N}$ we have $\Psi_{k}(F)=f^{(k)} / f$.

Bergweiler and Langley then prove the following theorem. For background material regarding Nevanlinna theory, the reader is referred to [5].

Theorem A ([1]) Let $k \geq 3$ be an integer, and let $F$ be a meromorphic and non-constant function in the plane that satisfies both of the following conditions:

(i) $\Psi_{k}(F)$ has no zeros.

(ii) if a is a simple pole of $F$ then $\operatorname{Res}(F, a) \notin\{1, \ldots, k-1\}$.

Then $F$ has the form

$$
F(z)=\frac{(k-1) z+\alpha}{z^{2}+\beta z+\gamma}
$$

or

$$
F(z)=\frac{1}{\alpha z+\beta} .
$$

\footnotetext{
${ }^{1}$ Department of Mathematics, University of Nottingham, Nottingham, NG7 2RD, U.K.

Email: eleanor.clifford@maths.nottingham.ac.uk
} 
Here $\alpha, \beta, \gamma \in \mathbb{C}$ with $\alpha \neq 0$ in (3).

Conversely, if $F$ has the form (2) or (3), and if $(i i)$ holds, then $\Psi_{k}(F)$ has no zeros. If $F$ has the form (2) or (3), but (ii) does not hold, then $\Psi_{k}(F) \equiv 0$.

We note that the conclusion of this theorem can be summarised as saying that $F$ is a rational function, of a special form, and hence,

$$
T(r, F)=O(\log r) \quad \text { as } r \rightarrow \infty \text {. }
$$

Defining an annulus $\mathcal{A}\left(r_{0}\right)$ by

$$
\mathcal{A}\left(r_{0}\right)=\left\{z: r_{0} \leq|z|<\infty\right\}
$$

we extend Theorem 1 in two ways. First, we let $F$ be meromorphic and non-constant on $\mathcal{A}\left(r_{0}\right)$, by which we mean that $F$ is meromorphic in a domain containing $\mathcal{A}\left(r_{0}\right)$. Second, we weaken condition $(i)$ as follows. We let $a_{0}, \ldots, a_{k-1}$ be analytic functions of restricted growth as $z \rightarrow \infty$, and define $\Lambda_{k}(F)$ by

$$
\Lambda_{k}(F)=\Psi_{k}(F)+a_{k-1} \Psi_{k-1}(F)+\ldots+a_{1} \Psi_{1}(F)+a_{0} .
$$

We then assume that $\Lambda_{k}(F)=0$ only on a set $E$ such that $E$ has no limit point in the annulus $\mathcal{A}\left(r_{0}\right)$. This implies that $\Lambda_{k}(F)=0$ only on a countable set $E$. The new conclusion is that $F$ is a function of restricted growth in the Nevanlinna sense. We state the extended theorem as follows, denoting by $S(r, F)$ any quantity satisfying

$$
S(r, F)=O\left(\log r+\log ^{+} T(r, F)\right),
$$

as $r \rightarrow \infty$ outside a set of finite measure, not necessarily the same set at each occurrence.

Theorem 1.2 Let $k \geq 3$ be an integer and let $F$ be meromorphic and non-constant in an annulus $\mathcal{A}\left(r_{0}\right)$, as defined by (4). Suppose $a_{0}, \ldots, a_{k-1}$ are analytic functions on $\mathcal{A}\left(r_{0}\right)$, with

$$
a_{j}(z)=O\left(|z|^{(\lambda-1)(k-j)}\right) \quad \text { as } z \rightarrow \infty,
$$

for some fixed $\lambda \geq 0$. Let $f_{1}, \ldots, f_{k}$ be solutions of $L(w)=0$ where $L$ is defined by

$$
L(f)=f^{(k)}+a_{k-1} f^{(k-1)}+\ldots+a_{0} f,
$$

in $\mathcal{A}\left(r_{0}\right) \backslash \mathbb{R}^{-}$. Let $\Lambda_{k}(F)$ be defined as in (5) by

$$
\Lambda_{k}(F)=\Psi_{k}(F)+a_{k-1} \Psi_{k-1}(F)+\ldots+a_{1} \Psi_{1}(F)+a_{0} .
$$

Suppose there exists a set $E$, such that $E$ has no limit point in $\mathcal{A}\left(r_{0}\right)$, and such that $\Lambda_{k}(F)$ has all its zeros in E. Suppose further that all simple poles a of $F$ in $\mathcal{A}\left(r_{0}\right) \backslash E$ have $\operatorname{Res}(F, a) \notin\{1, \ldots, k-1\}$. Set

$$
N_{E}(r)=\int_{r_{0}}^{r} \frac{n_{E}(t)}{t} d t
$$

where $n_{E}(t)$ is the number of points in $E \cap\left\{z: r_{0} \leq|z| \leq t\right\}$.

Then either:

(i) $T(r, F) \leq c N_{E}(r)+S(r, F)$, as $r \rightarrow \infty$, where $c$ is a constant depending only on $k$,

or

(ii) $F$ is a rational function of the $f_{j}$ and their derivatives, in which case

$$
T(r, F)=O\left(r^{\lambda}+\log r\right), \quad \text { as } r \rightarrow \infty .
$$

We note that when $\lambda=0$, it follows from (ii) that $\lim _{z \rightarrow \infty} F(z)$ exists.

The following corollaries are deduced from Theorem 1.2. 
Corollary 1.3 Let $k \geq 3$ be an integer and let $F$ be meromorphic and non-constant in an annulus $\mathcal{A}\left(r_{0}\right)$, as defined by (4). Suppose there exists a set $E$, such that $E$ has no limit point in $\mathcal{A}\left(r_{0}\right)$, and such that $\Psi_{k}(F)$ has all its zeros in $E$. Suppose further that all simple poles a of $F$, such that a $\notin E$, have $\operatorname{Res}(F, a) \notin$ $\{1, \ldots, k-1\}$. Then either:

(i) $T(r, F) \leq c N_{E}(r)+O\left(\log r+\log ^{+} T(r, F)\right) \quad$ (n.e.), where $c$ is a constant depending only on $k$,

or

(ii) $\lim _{z \rightarrow \infty} F(z)$ exists.

Corollary 1.4 Let $k \geq 3$ be an integer, and let $F$ be meromorphic and non-constant in an annulus $\mathcal{A}\left(r_{0}\right)$, as defined by (4). Suppose F satisfies both of the following conditions:

(i) $\Psi_{k}(F)$ has no zeros.

(ii) if a is a simple pole of $F$ then $\operatorname{Res}(F, a) \notin\{1, \ldots, k-1\}$.

Then $\lim _{z \rightarrow \infty} F(z)$ exists.

Corollary 1.5 Let $F$ be meromorphic on $\mathbb{C}$ and satisfy both of the following conditions:

(i) $\Psi_{k}(F)$ has finitely many zeros.

(ii) for all but finitely many simple poles of $F$ we have $\operatorname{Res}(F, a) \notin\{1, \ldots, k-1\}$.

Then $F$ is a rational function.

The following example shows that we cannot extend Theorem 1.2 to the case where $a_{0}, \ldots a_{k-1}$ are meromorphic functions.

Example Let $k \geq 3$ and let $F=f^{\prime} / f$ where $f$ is a meromorphic function which is nonvanishing, that is, has no zeros. Define $a_{0}, \ldots, a_{k-1}$ by

$$
a_{j}= \begin{cases}-\frac{f^{(k)}}{f} & \text { if } j=0, \\ \frac{f}{f^{(j)}} & \text { if } j=1, \ldots, k-1 .\end{cases}
$$

Then $a_{0}, \ldots, a_{k-1}$ are meromorphic functions and we have, using Lemma 1.1, that

$$
\begin{aligned}
\Lambda_{k}(F) & =\Psi_{k}(F)+a_{k-1} \Psi_{k-1}(F)+\ldots+a_{1} \Psi_{1}(F)+a_{0} \\
& =\frac{f^{(k)}}{f}+\frac{f}{f^{(k-1)}} \frac{f^{(k-1)}}{f}+\ldots+\frac{f}{f^{\prime}} \frac{f^{\prime}}{f}-\frac{f^{(k)}}{f} \\
& =k-1
\end{aligned}
$$

Thus $\Lambda_{k}(F)$ has no zeros, and $E=\emptyset$. And so, all the hypotheses of Theorem 1.2 are satisfied except for the analyticity and growth of the $a_{j}$. However, since $f$ may be any nonvanishing meromorphic function, no conclusions may be drawn about the growth of $F$.

This work was carried out as part of a Ph.D. thesis, and the author would like to thank J.K. Langley for his advice and encouragement.

Some preliminaries for the proof of Theorem 1.2 are in $\S 2$, and the main part of the proof is in $\S 3$. 


\section{Proof of Theorem 1.2 (Preliminaries)}

First we include some background material about Wronskians, referring the reader to [7] for further material. Let $f_{1}, \ldots, f_{k}$ be meromorphic functions. We define the Wronskian $W\left(f_{1}, \ldots, f_{k}\right)$ as follows

$$
W\left(f_{1}, \ldots, f_{k}\right)=\left|\begin{array}{ccc}
f_{1} & \ldots & f_{k} \\
f_{1}^{\prime} & \ldots & f_{k}^{\prime} \\
\vdots & & \vdots \\
f_{1}^{(k-1)} & \ldots & f_{k}^{(k-1)}
\end{array}\right|
$$

Following the notation in [7], we denote by $W_{q}\left(f_{1}, \ldots, f_{k}\right)$, for $q=0, \ldots, k-1$, the determinant which is obtained from $W\left(f_{1}, \ldots, f_{k}\right)$ by replacing the row $\left(f_{1}^{(q)}, \ldots, f_{k}^{(q)}\right)$ by $\left(f_{1}^{(k)}, \ldots, f_{k}^{(k)}\right)$. We include two useful lemmas regarding Wronskians, the second of which combines results from [4] and [7].

Lemma $2.1([7])$ Let $f_{1}, \ldots, f_{k}$ be meromorphic functions in a domain $\Omega$. Then $W\left(f_{1}, \ldots, f_{k}\right)$ vanishes identically on $\Omega$, if and only if $f_{1}, \ldots, f_{k}$ are linearly dependent on $\Omega$.

Lemma $2.2([4],[7])$ Let $k \geq 1$ and let $f_{1}, \ldots, f_{k}$ be linearly independent meromorphic functions in a domain $\Omega$, that satisfy the homogeneous linear differential equation $L(w)=0$, where $L$ is defined by $(7)$, and in which the $a_{q}$ are meromorphic on $\Omega$. Then the $a_{q}$ can be written in the form

$$
a_{q}=-\frac{W_{q}\left(f_{1}, \ldots, f_{k}\right)}{W\left(f_{1}, \ldots, f_{k}\right)}
$$

for $q=0, \ldots, k-1$ and, in particular,

$$
a_{k-1}=-W\left(f_{1}, \ldots, f_{k}\right)^{\prime} / W\left(f_{1}, \ldots, f_{k}\right) .
$$

The poles of $a_{q}$ in $\Omega$ have multiplicity $\leq k-q$ and can only arise among the poles of $f_{1}, \ldots, f_{k}$ and the zeros of $W\left(f_{1}, \ldots, f_{k}\right)$. Furthermore, if $f$ is meromorphic on $\Omega$ then

$$
W\left(f_{1}, \ldots, f_{k}, f\right)=W\left(f_{1}, \ldots, f_{k}\right) L(f) .
$$

Several lemmas are needed for the proof of Theorem 1.2. We state them here, providing proofs where necessary. The first assertion in the following lemma is proved in [1], and the second is an extension which follows immediately.

Lemma 2.3 ([1]) Let $k \geq 2$ be an integer. Let $y$ be meromorphic on a domain $\Omega$, such that if a is a simple pole of $y$ then $\operatorname{Res}(y, a) \notin\{1, \ldots, k-1\}$. Let $n \in \mathbb{N}$ be such that $n \leq k$. If $y$ has a pole at a of multiplicity $m$ then $\Psi_{n}(y)$ has a pole at a of multiplicity $n m$, and $\Lambda_{n}(y)$ has a pole at a of multiplicity nm, where $\Lambda_{n}(y)$ is defined as in (5) by

$$
\Lambda_{n}(y)=\Psi_{n}(y)+a_{n-1} \Psi_{n-1}(y)+\ldots+a_{1} \Psi_{1}(y)+a_{0}
$$

where $a_{0}, \ldots, a_{n-1}$ are analytic functions on $\Omega$.

The following summarises some results from Nevanlinna theory which are used in Lemma 2.8. For background material about Nevanlinna theory in an annulus, we refer the reader to [2] or [4].

Lemma 2.4 Let $p \in \mathbb{N}$ and let $\mathcal{B}$ be a simply connected domain in the annulus $\mathcal{A}\left(r_{0}\right)$, as defined by (4). Suppose that $w$ is meromorphic on $\mathcal{B}$ such that $W=w^{p}$ is meromorphic on $\mathcal{A}\left(r_{0}\right)$. Then $w^{(k)} / w$ is meromorphic on $\mathcal{A}\left(r_{0}\right)$, for each $k \in \mathbb{N}$, with poles of multiplicity at most $k$. Further, we have

$$
T\left(r, w^{(k)} / w\right)=O(T(r, W)), \quad(\text { n.e. }),
$$

and

$$
m\left(r, w^{(k)} / w\right)=S(r, W), \quad \text { (n.e.) }
$$

as $r \rightarrow \infty$. 
Proof For $k=1$ we note that $\frac{w^{\prime}}{w}=\frac{1}{p} \frac{W^{\prime}}{W}$, and the result follows. For $k \geq 2$, the result is easily proved by induction.

Next we state a lemma from [4].

Lemma 2.5 ([4]) Suppose that $f_{1}, \ldots, f_{k}$ each admit unrestricted analytic continuation in an annulus $\mathcal{A}\left(r_{0}\right)$, as defined by (4), and satisfy $\log ^{+} \log ^{+}\left|f_{j}(z)\right|=O(\log |z|)$ for $z$ in $\mathcal{S}=\left\{z:|z|>r_{0},|\arg z|<2 \pi\right\}$. Suppose that $F$ is meromorphic in $\mathcal{A}\left(r_{0}\right)$. Suppose further that, for some non-negative integer $Q$, each of the functions $h_{1}, \ldots, h_{k}$ on $\mathcal{S}$ is a polynomial in the $f_{j}^{(m)}, F^{(m)}, 1 \leq j \leq k, 0 \leq m \leq Q$. Suppose finally that $h_{1}, \ldots, h_{k}$ are linearly independent solutions in $\mathcal{S}$ of an equation

$$
w^{(k)}+\sum_{j=0}^{k-1} B_{j} w^{(j)}=0
$$

in which the $B_{j}$ are meromorphic in $\mathcal{A}\left(r_{0}\right)$. Then we have, for $j=0, \ldots, k-1$,

$$
m\left(r, B_{j}\right)=S(r, F) .
$$

The following lemma is proved in [4] for $a_{0}, \ldots, a_{k-1}$ rational functions, and $F$ meromorphic in the plane. The proof extends without modification to the case where $a_{0}, \ldots, a_{k-1}$ are analytic functions and $F$ is meromorphic in $\mathcal{A}\left(r_{0}\right)$. This gives the following lemma.

Lemma 2.6 Let $\lambda \geq 0$ and $k \geq 2$, and let $a_{0}, \ldots, a_{k-1}$ be analytic functions satisfying $a_{j}(z)=O\left(|z|^{(\lambda-1)(k-j)}\right)$ as $z \rightarrow \infty$. Suppose that $F$ is meromorphic in the annulus $\mathcal{A}\left(r_{0}\right)$, as defined by $(4)$, and has in some domain $\mathcal{B}$ a representation as a rational function in solutions $f_{j}$ of the equation $L(w)=0$ and their derivatives, where $L$ is defined by (7). If $\lambda>0$ then $T(r, F)=O\left(r^{\lambda}\right)$ as $r \rightarrow \infty$. If $\lambda=0$ then $\lim _{z \rightarrow \infty} F(z)$ exists.

The following lemma is found in [8].

Lemma 2.7 ([8]) Suppose that $k \geq 1$ and that $a_{0}, \ldots, a_{k-1}$ are analytic in an annulus $\mathcal{A}\left(r_{0}\right)$, as defined by (4), such that, for some $\lambda \geq 0$,

$$
a_{j}(z)=O\left(|z|^{(\lambda-1)(k-j)}\right), \quad \text { as } z \rightarrow \infty .
$$

Let $f_{j}(z)$ be a solution of $L(w)=0$, where $L$ is defined by $(7)$, in a sectorial region

$$
\mathcal{S}=\left\{z:|z|>r_{0}, \alpha<\arg z<\alpha+2 \pi\right\},
$$

where $\alpha$ is real. Then as $z \rightarrow \infty$ in $\mathcal{S}$,

$$
\log ^{+}\left|f_{j}(z)\right|=O\left(|z|^{\lambda}+\log |z|\right)
$$

Suppose now that $k, F, \mathcal{A}\left(r_{0}\right)$ and $a_{0}, \ldots, a_{k-1}$ are as in the statement of Theorem 1.2. In particular, the $a_{j}$ satisfy (11). We may define linearly independent analytic solutions $f_{1}, \ldots, f_{k}$ of $(7)$ in $\mathcal{A}\left(r_{0}\right) \backslash \mathbb{R}^{-}$. These are analytic in $\mathcal{A}\left(r_{0}\right) \backslash \mathbb{R}^{-}$and since the $a_{j}$ are analytic in $\mathcal{A}\left(r_{0}\right)$, the $f_{j}$ admit unrestricted analytic continuation in $\mathcal{A}\left(r_{0}\right)$. By $(12), \log ^{+}\left|f_{j}(z)\right|=O\left(|z|^{\lambda}+\log |z|\right)$ for $j=1, \ldots, k$ and thus the continuations satisfy $\log ^{+} \log ^{+}\left|f_{j}(z)\right|=O(\log |z|)$ for $|z|>r_{0},|\arg z|<2 \pi$. Then $f_{1}, \ldots, f_{k}$ satisfy the conditions of Lemma 2.5 .

Choose a simply connected domain $\mathcal{B} \subseteq \mathcal{A}\left(r_{0}\right)$, on which $F$ has no poles and $\Lambda_{k}(F)$ has no zeros. Define functions $f, g$, and $h$ in $\mathcal{B}$ by

$$
f^{\prime} / f=F, \quad \Lambda_{k}(F)=g^{-k}, \quad h=-F g .
$$

Then $f, g$ and $h$ are analytic on $\mathcal{B}$.

We note that by Lemma 1.1 , we have in $\mathcal{B}$ that

$$
L(f) / f=f^{(k)} / f+\sum_{j=0}^{k-1} a_{j} f^{(j)} / f=\Psi_{k}(F)+\sum_{j=1}^{k-1} a_{j} \Psi_{j}(F)+a_{0}=\Lambda_{k}(F) .
$$

The following lemma is fundamental to our proof of Theorem 1.2 and uses ideas such as analytic continuation and Wronskians. Background material on these topics can be found in [3] and [7]. 
Lemma 2.8 Define on $\mathcal{B}$ functions $w_{j}$ and $h_{j}, j=1, \ldots, k$, by

$$
w_{j}(z)=f_{j}^{\prime}(z) g(z)+f_{j}(z) h(z), \quad h_{j}(z)=-f_{j}^{\prime}(z)+f_{j}(z) F(z) .
$$

Then the $w_{j}$ form a fundamental solution set on $\mathcal{B}$ of the differential equation

$$
w^{(k)}+\sum_{j=0}^{k-1} A_{j} w^{(j)}=0,
$$

in which the $A_{j}$ are meromorphic functions on $\mathcal{A}\left(r_{0}\right)$ with

$$
T\left(r, A_{q}\right) \leq c N_{E}(r)+S(r, F), \quad r \rightarrow \infty,
$$

for $j=0, \ldots, k-1$, where $c$ is a constant depending only on $k$.

Proof We divide this proof into a number of steps.

(i) The $w_{j}$ are linearly independent solutions of an equation (16) on $\mathcal{B}$.

We note first that $w_{j}=f g\left(f_{j} / f\right)^{\prime}$ on $\mathcal{B}$ by (13), and by (10) and the properties of Wronskians we have

$$
W\left(w_{1}, \ldots, w_{k}\right)=f^{-1} g^{k}(-1)^{k} W\left(f_{1}, \ldots, f_{k}, f\right)=f^{-1} g^{k}(-1)^{k} L(f) W\left(f_{1}, \ldots, f_{k}\right) .
$$

Then since $L(f) / f=\Lambda_{k}(F)$ by (14), and $g^{k}=\left(\Lambda_{k}(F)\right)^{-1}$ by (13), we have from (18) that,

$$
W\left(w_{1}, \ldots, w_{k}\right)=(-1)^{k} W\left(f_{1}, \ldots, f_{k}\right) .
$$

By Lemma 2.1, the right-hand side is not identically zero, since the $f_{j}$ form a linearly independent solution set of (7). Thus $w_{1}, \ldots, w_{k}$ form a linearly independent solution set for a differential equation (16). Also, by (9), we have

$$
A_{k-1}=-\frac{W\left(w_{1}, \ldots, w_{k}\right)^{\prime}}{W\left(w_{1}, \ldots, w_{k}\right)}=-\frac{(-1)^{k} W\left(f_{1}, \ldots, f_{k}\right)^{\prime}}{(-1)^{k} W\left(f_{1}, \ldots, f_{k}\right)}=a_{k-1} .
$$

(ii) The $h_{j}$ are linearly independent solutions of a differential equation,

$$
w^{(k)}+\sum_{j=0}^{k-1} B_{j} w^{(j)}=0
$$

with coefficients $B_{j}$ that are meromorphic on $\mathcal{A}\left(r_{0}\right) \backslash \mathbb{R}^{-}$.

We have by (13) and (15) that $w_{j}=-h_{j} g$, and so

$$
W\left(w_{1}, \ldots, w_{k}\right)=(-1)^{k} g^{k} W\left(h_{1}, \ldots, h_{k}\right)=(-1)^{k} \Lambda_{k}(F)^{-1} W\left(h_{1}, \ldots, h_{k}\right) .
$$

Then by (19), we have

$$
W\left(h_{1}, \ldots, h_{k}\right)=\Lambda_{k}(F) W\left(f_{1}, \ldots, f_{k}\right) .
$$

The right-hand side is not identically zero on $\mathcal{B}$ since $\Lambda_{k}(F) \neq 0$ on $\mathcal{B}$ and since $f_{1}, \ldots, f_{k}$ form a linearly independent solution set to $(7)$ on $\mathcal{A}\left(r_{0}\right) \backslash \mathbb{R}^{-}$. Thus, $h_{1}, \ldots, h_{k}$ form a linearly independent solution set to a differential equation (21) on $\mathcal{B}$. By (15), we have $h_{j}=-f_{j}^{\prime}+f_{j} F$ and thus the $h_{1}, \ldots, h_{k}$ are meromorphic on $\mathcal{A}\left(r_{0}\right) \backslash \mathbb{R}^{-}$since the $f_{j}$ are analytic there, and $F$ is meromorphic on $\mathcal{A}\left(r_{0}\right)$. Hence the coefficients $B_{j}$ are meromorphic on $\mathcal{A}\left(r_{0}\right) \backslash \mathbb{R}^{-}$.

(iii) The $B_{j}$ extend to be meromorphic on $\mathcal{A}\left(r_{0}\right)$ and have poles of multiplicity $\leq k-j$ on $\mathcal{A}\left(r_{0}\right)$.

By analytic continuation of the $f_{j}$, the $B_{j}$ extend to be meromorphic on $\mathcal{A}\left(r_{0}\right)$. Furthermore, $(21)$ has $k$ linearly independent solutions on a neighbourhood of each point of $\mathcal{A}\left(r_{0}\right)$, namely $h_{1}, \ldots, h_{k}$. Hence, by Lemma 2.2 , the $B_{j}$ have poles of multiplicity $\leq k-j$ on $\mathcal{A}\left(r_{0}\right)$. 
(iv) Estimate for $m\left(r, A_{j}\right)$.

First we recall by (15) that $h_{j}=-f_{j}^{\prime}+f_{j} F$, and note that all the conditions of Lemma 2.5 are satisfied, and thus we have that

$$
m\left(r, B_{j}\right)=S(r, F)
$$

for $j=0, \ldots, k-1$. Next expressing $A_{j}$ in terms of $B_{j}$ and $g^{(p)} / g$, we have for $j=0, \ldots, k-1$,

$$
A_{j}=B_{j}-\left(\begin{array}{l}
k \\
j
\end{array}\right) \frac{g^{(k-j)}}{g}-\sum_{q=j+1}^{k-1} A_{q}\left(\begin{array}{l}
q \\
j
\end{array}\right) \frac{g^{(q-j)}}{g},
$$

which is initialised by

$$
A_{k-1}=B_{k-1}-\left(\begin{array}{c}
k \\
k-1
\end{array}\right) \frac{g^{\prime}}{g} .
$$

We now note by Lemma 2.4 that $g^{(p)} / g$ is meromorphic in $\mathcal{A}\left(r_{0}\right)$ for all $p \in \mathbb{N}$, and that $m\left(r, g^{(p)} / g\right)=S(r, F)$ for $p=1, \ldots, k$. Thus we have by (22) and by induction on (23), that

$$
m\left(r, A_{j}\right)=S(r, F)
$$

for $j=0, \ldots, k-1$.

(v) Estimate for $N\left(r, A_{j}\right)$.

We show first that the poles of $A_{j}$ can only arise on $E$, the set containing all points where $\Lambda_{k}(F)=0$.

We know by Lemma 2.2, that the poles of $A_{j}$ can only arise among the zeros of the continuation of $W\left(w_{1}, \ldots, w_{k}\right)$ and the poles of the continuations of $w_{1}, \ldots, w_{k}$. By $(20), a_{k-1}=-W\left(w_{1}, \ldots, w_{k}\right)^{\prime} / W\left(w_{1}, \ldots, w_{k}\right)$ and since $a_{k-1}$ is analytic on $\mathcal{A}\left(r_{0}\right)$, we have that $W\left(w_{1}, \ldots, w_{k}\right)$ continues without zeros. We recall from (13) and 15) that $w_{j}=\left(f_{j}^{\prime}-f_{j} F\right) /\left(\Lambda_{k}(F)\right)^{1 / k}$. Thus the poles of $A_{j}$ only arise at poles of $F$ and zeros of $\Lambda_{k}(F)$.

Now let $z_{0} \in \mathcal{A}\left(r_{0}\right) \backslash E$ and suppose that a pole of $A_{j}$ arises at $z_{0}$. Since $\Lambda_{k}(F) \neq 0$ on $\mathcal{A}\left(r_{0}\right) \backslash E$ we must have that $z_{0}$ is a pole of $F$, of multiplicity $m$ say, and if $m=1$ then $\operatorname{Res}\left(F, z_{0}\right) \notin\{1, \ldots, k-1\}$ since $F$ satisfies the hypotheses of Theorem 1.2. Then by Lemma $2.3, \Lambda_{k}(F)$ has a pole at $z_{0}$ of multiplicity $m k$, and so $g$ can be analytically continued to a neighbourhood of $z_{0}$ and has a zero of multiplicity $m$ there. Thus $h=-F g$ can be analytically continued to $z_{0}$ and since the $f_{j}$ can be continued analytically in $\mathcal{A}\left(r_{0}\right)$, we have that $w_{j}=f_{j}^{\prime} g+f_{j} h$ can be analytically continued to $z_{0}$. We therefore deduce that the $A_{j}$ are analytic at $z_{0}$. This contradicts our hypothesis, and so the poles of $A_{j}$ can only arise in $E$.

We recall from (iv) that the poles of $B_{j}$ have multiplicity $\leq k-j$ on $\mathcal{A}\left(r_{0}\right)$. We note also by Lemma 2.4 , that $g^{(p)} / g$ have poles of multiplicity $\leq p$ there. Thus we have by (23) that the poles of $A_{j}$ must have multiplicity $\leq c$ where $c$ is a constant depending only on $k$. Therefore we have, for $j=0, \ldots, k-1$,

$$
N\left(r, A_{j}\right) \leq c N_{E}(r) .
$$

(vi) Conclusion.

We have since $T\left(r, A_{j}\right)=N\left(r, A_{j}\right)+m\left(r, A_{j}\right)$ that

$$
T\left(r, A_{j}\right) \leq c N_{E}(r)+S(r, F), \quad \text { as } r \rightarrow \infty,
$$

for $j=0, \ldots, k-1$, where $c$ is a constant depending only on $k$. This completes the proof of Lemma 2.8.

The following lemma appears in [4] and is the final lemma needed for the main part of the proof of Theorem 1.2 .

Lemma 2.9 ([4]) Let $k \geq 1$ be an integer, and let $f_{1}, \ldots, f_{k}, G, H$ and $a_{0}, \ldots, a_{k-1}$ and $A_{0}, \ldots, A_{k-1}$ be meromorphic in a domain $\Omega$. Suppose that $f_{1}, \ldots, f_{k}$ are linearly independent solutions in $\Omega$ of $L(w)=0$, where $L$ is defined as in (7). Then the functions $f_{1}^{\prime} g+f_{1} h, \ldots, f_{k}^{\prime} g+f_{k} h$ are solutions in $\Omega$ of the equation (16) if and only if, setting $A_{k}=1$ and $A_{-1}=a_{-1}=0$ and, for $0 \leq q \leq k$,

$$
M_{k, q}(w)=\sum_{m=q}^{k}\left(\begin{array}{c}
m \\
q
\end{array}\right) A_{m} w^{(m-q)}, \quad M_{k,-1}(w)=0,
$$


we have, for $0 \leq q \leq k-1$,

$$
M_{k, q}(h)-a_{q} h=-M_{k, q-1}(g)+a_{q} M_{k, k-1}(g)-\left(a_{q} a_{k-1}-a_{q}^{\prime}-a_{q-1}\right) g .
$$

\section{Proof of Theorem 1.2 (Main Part)}

We are now in a position to complete the proof of Theorem 1.2. We note that we use methods found in [4, Theorem 3].

Proof of Theorem 1.2 We apply Lemma 2.9 to equation (16) and to $g$ and $h$ in $\mathcal{B}$. The $k$ equations (24) can be written in the form

$$
T_{q}(g)=S_{q}(h)=\sum_{j=0}^{k-q} c_{j, q} h^{(j)}, \quad 0 \leq q \leq k-1,
$$

in which $T_{q}$ and $S_{q}$ are homogeneous linear differential operators with coefficients $\lambda_{\nu}$ which are rational functions in the $a_{j}, A_{j}$ and their derivatives. Then by (17) we have

$$
T\left(r, \lambda_{\nu}\right) \leq c N_{E}(r)+S(r, F), \quad \text { as } r \rightarrow \infty,
$$

where $c$ is a constant depending only on $k$.

We have in particular that $q=k-1$ gives

$$
M_{k, k-1}(h)-a_{k-1} h=-M_{k, k-2}(g)+a_{k-1} M_{k, k-1}(g)-\left(a_{k-1} a_{k-1}-a_{k-1}^{\prime}-a_{k-2}\right) g .
$$

Then since

$$
\begin{aligned}
& M_{k, k-1}(h)=A_{k-1} h+k h^{\prime} \\
& M_{k, k-1}(g)=A_{k-1} g+k g^{\prime} \\
& M_{k, k-2}(g)=A_{k-2} g+(k-1) A_{k-1} g^{\prime}+k(k-1) g^{\prime \prime} / 2
\end{aligned}
$$

we now have that

$$
\begin{aligned}
A_{k-1} h+k h^{\prime}-a_{k-1} h= & -A_{k-2} g-(k-1) A_{k-1} g^{\prime}-k(k-1) g^{\prime \prime} / 2+a_{k-1} A_{k-1} g+ \\
& +k a_{k-1} g^{\prime}-\left(a_{k-1} a_{k-1}-a_{k-1}^{\prime}-a_{k-2}\right) g
\end{aligned}
$$

which gives

$$
h^{\prime}=U(g)=-(k-1) g^{\prime \prime} / 2+a_{k-1} g^{\prime} / k+\left(a_{k-1}^{\prime}+a_{k-2}-A_{k-2}\right) g / k
$$

since $a_{k-1}=A_{k-1}$ on $\mathcal{B}$ by (20). We note that we can then write (25) in the form

$$
T_{q}(g)=c_{0, q} h+\sum_{j=1}^{k-q} c_{j, q} \frac{d^{j-1}}{d z^{j-1}}(U(g)) .
$$

We distinguish two cases here.

Case 1. We assume that the coefficient of $h$ in at least one $S_{q}$ in (25) is not identically zero.

Let $\nu$ be the largest integer, $0 \leq \nu \leq k-1$, such that $c_{0, \nu} \not \equiv 0$. Then since $h=-F g$ by (13), equations (25) and (28) give

$$
h=-F g=\left(c_{0, \nu}\right)^{-1}\left(T_{\nu}(g)-\sum_{j=1}^{k-\nu} c_{j, \nu} \frac{d^{j-1}}{d z^{j-1}}(U(g))\right)=V(g) .
$$

Then by (25), (27) and (29) we know that $g$ solves the system of equations

$$
U(g)=\frac{d}{d z}(V(g)), \quad S_{q}(V(g))=T_{q}(g), \quad 0 \leq q \leq k-2 .
$$


Here we distinguish two sub-cases.

Case 1A. We assume that the solution space of (30) has dimension 1 . That is, every common solution of the equations (30) is a constant multiple of $g$.

Then (26) and a standard reduction procedure, see [6, p.126], give a first order equation

$$
p_{1} g^{\prime}+p_{0} g=0, \quad p_{1} \not \equiv 0,
$$

where the $p_{j}$ are rational functions in the $\lambda_{\nu}$ and their derivatives. It follows by (26) that

$$
T\left(r, g^{\prime} / g\right) \leq c N_{E}(r)+S(r, F), \quad \text { as } r \rightarrow \infty,
$$

where $c$ is a constant depending only on $k$. Hence, since $F=-h / g$ and using (26) and (29),

$$
T(r, F) \leq c N_{E}(r)+S(r, F), \quad \text { as } r \rightarrow \infty .
$$

Hence we have conclusion $(i)$ of the theorem.

Case 1B. We assume that there is a solution $G$ for the system (30) such that $G / g$ is non-constant. In particular, we note that this will be the case if the system (30) is trivial.

Define $H$ by $H=V(G)$. Then, by $(30)$,

$$
H^{\prime}=U(G), \quad S_{q}(H)=T_{q}(G), \quad 0 \leq q \leq k-2 .
$$

In particular, the equations (25) hold with $g$ and $h$ replaced by $G$ and $H$ respectively. And so, by Lemma 2.9 , the functions $f_{j} H+f_{j}^{\prime} G$ are solutions of (16) and so are linear combinations of $w_{1}, \ldots, w_{k}$. Hence, there are solutions $g_{j}$ of $L(f)=0$ where $L$ is defined by (7) such that

$$
f_{j} H+f_{j}^{\prime} G-g_{j} h-g_{j}^{\prime} g=0, \quad 1 \leq j \leq k .
$$

We regard the equations in (31) as a system of $k$ equations in $H, G, h, g$, over the field $\mathbb{F}$ of functions meromorphic in $\mathcal{B}$, with coefficients $f_{j}, f_{j}^{\prime}, g_{j}, g_{j}^{\prime}$.

Next, we note that the rank of the coefficient matrix is $\leq 3$, since there is a non-trivial solution for the system. Using the method found in [4, Theorem 3], we have that the rank of the system (31) is precisely 3. We can then solve for $-F=h / g$ as a quotient of determinants in $f_{j}, f_{j}^{\prime}, g_{j}, g_{j}^{\prime}$. Thus $F$ is a rational function of the $f_{j}$ and their derivatives. By Lemma 2.6

$$
T(r, F)=O\left(r^{\lambda}+\log r\right), \quad \text { as } r \rightarrow \infty,
$$

and so we have conclusion $(i i)$ of the theorem.

Case 2. We assume that $c_{0, q} \equiv 0$ for $0 \leq q \leq k-1$ in $(25)$.

We then have that the equations (25) are satisfied when $g$ and $h$ are replaced by 0 and 1 respectively and thus so are the equations $(24)$. Then, by Lemma 2.9 , the $f_{j}$ are solutions of $(16)$. Thus the equations $L(f)=0$ and (16) are the same, where $L$ is defined by (7), and for $1 \leq q \leq k$ we may write

$$
f_{j} h+f_{j}^{\prime} g=g_{j},
$$

in which each $g_{j}$ is a solution of $L(f)=0$. Then since $f_{1}$ and $f_{2}$ are linearly independent, we have $f_{1} f_{2}^{\prime}-f_{1}^{\prime} f_{2} \not \equiv 0$ and so

$$
F=-h / g=\left(f_{1}^{\prime} g_{2}-f_{2}^{\prime} g_{1}\right) /\left(f_{1} g_{2}-f_{2} g_{1}\right),
$$

which gives $F$ as a quotient of determinants in $f_{j}, f_{j}^{\prime}, g_{j}, g_{j}^{\prime}$. Then by Lemma 2.6 we have that

$$
T(r, F)=O\left(r^{\lambda}+\log r\right), \quad \text { as } r \rightarrow \infty,
$$

and so we have conclusion $(i i)$ of the theorem.

This completes the proof of Theorem 1.2. 


\section{References}

[1] Walter Bergweiler and J. K. Langley. Nonvanishing derivatives and normal families. J. Anal. Math., 91:353-367, 2003.

[2] Ludwig Bieberbach. Theorie der gewöhnlichen Differentialgleichungen auf funktionentheoretischer Grundlage dargestellt. Die Grundlehren der mathematischen Wissenschaften in Einzeldarstellungen mit besonderer Berücksichtigung der Anwendungsgebiete, Bd LXVI. Springer-Verlag, Berlin, 1953.

[3] John B. Conway. Functions of one complex variable. Springer-Verlag, New York, 1973. Graduate Texts in Mathematics, 11.

[4] G. Frank and J. K. Langley. Pairs of linear differential polynomials. Analysis (Munich), 19(2):173-194, 1999.

[5] W. K. Hayman. Meromorphic functions. Oxford Mathematical Monographs. Clarendon Press, Oxford, 1964.

[6] E. L. Ince. Ordinary differential equations. Dover Publications, New York, 1944.

[7] Ilpo Laine. Nevanlinna theory and complex differential equations, volume 15 of de Gruyter Studies in Mathematics. Walter de Gruyter \& Co., Berlin, 1993.

[8] J. K. Langley. An application of the Tsuji characteristic. J. Fac. Sci. Univ. Tokyo Sect. IA Math., 38(2):299-318, 1991. 